\title{
Can roflumilast, a phosphodiesterase- 4 inhibitor, improve clinical outcomes in patients with moderate-to-severe chronic obstructive pulmonary disease? A meta- analysis
}

Jian Luo ${ }^{1+}$, Ke Wang ${ }^{1+}$, Dan Liu², Bin-Miao Liang ${ }^{1,3^{*+}}$ and Chun-Tao Liu ${ }^{1,3^{*+}}$

\begin{abstract}
Background: Effects of roflumilast on lung function, symptoms, acute exacerbation and adverse events in patients with chronic obstructive pulmonary disease (COPD) are controversial. We aimed to further clarify the efficacy and safety of roflumilast in treatment of moderate-to-severe COPD.

Methods: From 1946 to November 2015, we searched the Pubmed, Embase, Medline, Cochrane Central Register of Controlled Trials, ISI Web of Science and American College of Physician using "roflumilast" and "chronic obstructive pulmonary disease" or "COPD". Randomized controlled trials that reported forced expiratory volume in one second $\left(F E V_{1}\right)$, forced vital capacity $(F V C)$, transition dyspnea index (TDI), St George's Respiratory Questionnaire (SGRQ), and incidence of COPD exacerbations and adverse events were eligible. We conducted the heterogeneities test and sensitivity analysis, and random-effects or fixed-effects model was applied to calculate risk ratio (RR) and mean difference (MD) for dichotomous and continuous data respectively. Cochrane systematic review software, Review Manager (RevMan), was used to test the hypothesis by Mann-Whitney U-test.

Results: Thirteen trials with a total of 14,563 patients were pooled in our final studies. Except for SGRQ $\left(I^{2}=63 \%\right.$, $\left.X^{2}=1.71, P=0.07\right)$ and adverse events $\left(P^{2}=94 \%, X^{2}=0.03, P<0.001\right)$, we did not find statistical heterogeneity in outcome measures. The pooled MD of pre- and post-bronchodilator $\mathrm{FEV}_{1}$ was 54.60 (95\% confidence interval (Cl) $46.02 \sim 63.18)$ and $57.86(95 \% \mathrm{Cl} 49.80 \sim 65.91)$, and both showed significant improvement in patients with roflumilast $(z=12.47, P<0.001 ; z=14.07, P<0.001)$, so did in FVC (MD 90.37, $95 \%$ Cl 73.95 106.78, $z=10.79, P<0.001)$. Significant alleviation of TDI (MD 0.30, $95 \% \mathrm{Cl} 0.14 \sim 0.46, z=3.67, P<0.001$ ) and decrease of acute exacerbation (RR 0.86, $95 \% \mathrm{Cl} 0.81 \sim 0.91, z=5.54, P<0.001)$ were also identified in treatment of roflumilast, but without significant difference in SGRQ (MD $-1.30,95 \% \mathrm{Cl}-3.16 \sim 0.56, z=1.37, P=0.17)$. Moreover, roflumilast significantly increased the incidence of adverse events compared with placebo (RR 1.31, $95 \% \mathrm{Cl} 1.16 \sim 1.47, z=4.32, P<0.001)$.
\end{abstract}

Conclusions: Roflumilast can be considered as an alternative therapy in selective patients with moderate-to-severe COPD. Keywords: Roflumilast, Chronic obstructive pulmonary disease, Lung function, Exacerbation, Meta-analysis

\footnotetext{
* Correspondence: liangbinmiao@163.com; liuct0910@yahoo.com

${ }^{\dagger}$ Equal contributors

${ }^{1}$ Department of Respiratory and Critical Care Medicine, Sichuan University,

Chengdu, China

Full list of author information is available at the end of the article
}

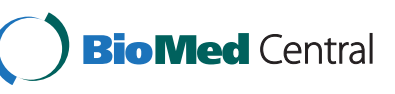

C 2016 Luo et al. Open Access This article is distributed under the terms of the Creative Commons Attribution 4.0 International License (http://creativecommons.org/licenses/by/4.0/), which permits unrestricted use, distribution, and reproduction in any medium, provided you give appropriate credit to the original author(s) and the source, provide a link to the Creative Commons license, and indicate if changes were made. The Creative Commons Public Domain Dedication waiver (http://creativecommons.org/publicdomain/zero/1.0/) applies to the data made available in this article, unless otherwise stated. 


\section{Background}

Chronic obstructive pulmonary disease (COPD), a common disease with a prevalence reported to be 7.8 to $19.7 \%$, is characterized by persistent and progressive airflow limitation as well as frequent exacerbations [1, 2]. Global Burden of Disease (GBD) Study projected that COPD will become the third leading cause of death worldwide by 2020, and it was estimated as the direct underlying cause of $7.8 \%$ of all deaths and $27 \%$ of deaths related with smoking $[3,4]$.

Acute exacerbation of COPD is defined as respiratory symptoms deterioration and medication alteration, and it has been demonstrated to be associated with detriment of quality of life, decline of lung function and increase of mortality [1, 5-7]. Hurst and his colleagues analyzed 2138 patients with COPD, and they found a trend of more exacerbations as the severity of COPD increased, that was 22, 33 and $47 \%$ in stage 2 (moderate), stage 3 (severe) and stage 4 (very severe), respectively [8]. Therefore, effective treatment and management in patients with moderate-to-severe COPD is paramount to decrease exacerbations, and improve lung function, quality of life and clinical outcomes.

Phosphodiesterase-4 (PDE4) is a vital enzyme in the metabolism of cyclic adenosine monophosphate (cAMP) and inhibition of PDE4 can inactivate immune and inflammatory cells via increase cAMP [9]. It is recommended by the Global Initiative for Chronic Obstructive Lung Disease (GLOD) guideline that a combination of PDE4 inhibitor and long-acting bronchodilator can be considered as an alternative treatment in patients with severe COPD due to the effective improvement of lung functions [1]. Roflumilast is a novel selective inhibitor of PDE4, which functions mainly by its active metabolite, roflumilast $\mathrm{N}$-oxide, via the conversion by cytochrome P450 (CYP) 3A4 and 1A2 isozymes [10]. Rabe and his colleagues conducted a randomized controlled trial (RCT) in 1157 patients with moderate-to-severe COPD, and they found that roflumilast could significantly improve post-bronchodilator forced expiratory volume in one second $\left(\mathrm{FEV}_{1}\right)(0.097 \pm$ $0.018, P<0.0001)$ and post-bronchodilator forced vital capacity (FVC) $(0.114 \pm 0.031, P=0.0002)$, and decrease incidence of acute exacerbations ( $28 \%$ vs. $35 \%, P=$ 0.0114) compared with placebo [11], which were further demonstrated by a subsequent meta-analysis of seven trials with 9675 patients but without improving healthrelated quality of life by St George's Respiratory Questionnaire (SGRQ) (mean difference (MD) -0.70, $95 \%$ confidence interval (CI) $-2.65 \sim 1.26, P=0.49$ ) or decreasing mortality rate (risk ratio (RR) 0.90, $95 \% \mathrm{CI}$ $0.63 \sim 1.29, P=0.56)$ [12].

However, Fabbri and his colleagues randomly assigned 743 patients with moderate-to-severe COPD into roflumilast plus tiotropium and tiotropium groups and they reported a significant improvement in Shortness of Breath Questionnaire (SOBQ) (MD -2.6, $95 \% \mathrm{CI}-4.5 \sim-0.8, P=$ 0.0051 ) in roflumilast plus tiotropium [13]. Moreover, a recent placebo-controlled randomized study, which investigated the additional treatment of roflumilast in moderate-to-severe COPD with chronic bronchitis, did not reveal any significant changes in lung function, quality of life, or exercise tolerance between rolumilast and placebo [14]. Therefore, the accurate roles of roflumilast in the treatment of patients with COPD still remain controversial.

In this study, we conducted a meta-analysis of all published RCTs with the aim of updating and further clarifying the efficacy and safety of roflumilast in patients with COPD.

\section{Methods}

Our study protocol was approved by the Institutional Ethical Committee for Clinical and Biomedical Research of West China Hospital (Sichuan, China), so did in each enrolled trial by the corresponding institutional review board. All participants provided written informed consent.

\section{Search strategies}

From 1946 to November 2015, a comprehensive computer search was conducted in Pubmed, Embase, Medline, Cochrane Central Register of Controlled Trials (CENTRAL), ISI Web of Science and American College of Physician (ACP) using the keywords of "roflumilast" and "chronic obstructive pulmonary disease" or "COPD" with limitation in the publication type of RCTs but not in the publication language. We reviewed the references listed in each identified article and manually searched the related articles to identify all eligible studies and minimize the potential publication bias.

\section{Inclusion and exclusion criteria}

Eligible clinical trials were defined based on the following criteria: 1) study design was RCT; 2) moderate-to-severe COPD was diagnosed by physicians according to the guidelines released by GOLD with a post-bronchodilator $\mathrm{FEV}_{1}$ between 30 and $80 \%[1]$; 3) age was more than 40 years old and smoking history was more than 10 packyears; and 4) intervention treatment was oral roflumilast with a dose of 500ug and a frequency of once daily, but regardless of administration durations. We did not enroll trials that were retrospective, observational, cohort or case control studies.

\section{Outcome measures}

Outcome measures consisted of efficacy assessment and safety evaluation, which included: 1) change of lung functions from baseline, such as pre-bronchodilator $\mathrm{FEV}_{1}$ and 
post-bronchodilator $\mathrm{FEV}_{1}$, FVC, force expiratory volume in six seconds $\left(\mathrm{FEV}_{6}\right)$ and forced expiratory flow between 25 and $75 \%$ of the vital capacity $\left.\left(\mathrm{FEF}_{25-75}\right) ; 2\right)$ healthrelated quality of life such as investigator-administered transition dyspnea index (TDI) and SGRQ, and 3) incidence of COPD exacerbations and adverse events.

\section{Study selection}

Two independent investigators performed the study selection in two phases. Firstly, they discarded duplicated and non-randomized controlled studies by screening titles and abstracts. Secondly, eligible studies were extracted by reviewing full texts in accordance with the previously designed study inclusion criteria. Any disagreement was solved by mutual consensus in the presence of a third investigator.

\section{Data extraction}

Independently, two data collectors extracted and recorded desirable information of each enrolled study in a standard form recommended by Cochrane [15], which consisted of authors, publication year, registration series, study design, participants and population, demographic characteristics (age, gender, etc.), baseline characteristics $\left(\mathrm{FEV}_{1} / \mathrm{FVC}\right.$, post-bronchodilator $\mathrm{FEV}_{1}$, post-bronchodilator $\mathrm{FEV}$, etc.), details of intervention treatment (dose, frequency, routine, and duration), follow-up period, and outcome measures and study results. For any missing data information, corresponding authors were contacted by email to request the full original data. Different opinions between the two collectors were determined by reaching a consensus or consulting a third investigator.

\section{Quality assessment}

For the assessment of risk of bias in estimating the study outcomes, we used the Cochrane risk of bias tool [15]. Each study was assessed for: 1) random sequence generation (selection bias); 2) allocation concealment (selection bias); 3) blinding of participants and personnel (performance bias); 4) blinding of related outcomes assessment (detection bias); 5) incomplete outcome data (attrition bias); 6) selective reporting (reporting bias); and 7) other biases. Two investigators conducted the quality assessment for the study methodology, independently and in duplicate. Any divergence was resolved by mutual consensus in the presence of a third investigator.

\section{Statistical analysis}

Statistical analysis of our study was accomplished by an independent statistician using Cochrane systematic review software Review Manager (RevMan; Version 5.3.5., The Nordic Cochrane Centre, The Cochrane Collaboration, Copenhagen, 2014). We used Mann-Whitney $U$-test to verify hypothesis and rendered statistical significance as $z$ - value and $P$-value $<0.05$, and the results were displayed in Forest plots.

Continuous variables were reported as mean and standard derivation (SD), while dichotomous variables were shown as frequency and proportion. An initial test for clinical, methodological and statistical heterogeneities was conducted, and we used the $\chi^{2}$ test with $P<0.1$ and $I^{2}>$ $50 \%$ to indicate significance. We also performed the sensitivity analysis to substitute alternative decisions or ranges of values for decisions that were arbitrary or unclear, and tested the publication biases by Funnel plot.

Random-effects model was applied in the presence of statistical heterogeneity; otherwise fixed-effects model was used. For continuous data we calculated MD and $95 \% \mathrm{CI}$, while for dichotomous data we calculated RR and $95 \%$ CI. Furthermore, in terms of pre- and post-bronchodilator $\mathrm{FEV}_{1}$, incidence of COPD exacerbation and adverse events, we separately conducted sub-analysis at different follow-up time points.

\section{Results}

Initially 120 records were identified, of which 118 were extracted from electronic databases and 2 were extracted from reference lists review. (Fig. 1) By screening the titles and abstracts, 99 studies were discarded for duplication $(n=52)$, not RCTs $(n=40)$, patients without COPD $(n=6)$, and intervention treatment without roflumilast $(n=1)$. We searched the full-text articles for the remaining 21 studies, and eventually 13 trials [11, 13, 14, 16-25] were enrolled in our final analysis due to 5 studies not reporting eligible outcomes and 3 studies being retrospective studies.

\section{Study description}

All 13 studies enrolled patients with severe COPD, but 2 studies $[16,17]$ also included mild COPD, 7 studies [11, $13,14,16,17,20,23]$ included moderate COPD, and 6 studies $[18,19,21,22,24,25]$ included very severe COPD. Except for one study [13] administering roflumilast plus salmeterol or tiotropium as intervention treatment and placebo plus salmeterol or tiotropium as control, all studies compared roflumilast with placebo. Treatment duration and follow-up period were not identical in different studies with 2 studies [20, 23] for 12 weeks, 1 study [14] for 14 weeks, 4 studies $[11,13,17,24]$ for 24 weeks, 1 study [16] for 26 weeks, and 5 studies [18, 19, 21, 22, 25] for 52 weeks. In terms of the outcome measures, 11 studies $[11,13,14,16-21,23,24]$ reported change of prebronchodilator $\mathrm{FEV}_{1}, 10$ studies [11, 13, 14, 18-21, 23-25] reported change of post-bronchodilator $\mathrm{FEV}_{1}, 8$ studies $[11,13,14,18-20,24,25]$ provided change of postbronchodilator FVC, 3 studies [11, 18, 20] provided change of post-bronchodilator $\mathrm{FEV}_{6}, 5$ studies [11, 18-20, 23] provided change of post-bronchodilator $\mathrm{FEF}_{25-75}, 4$ studies 


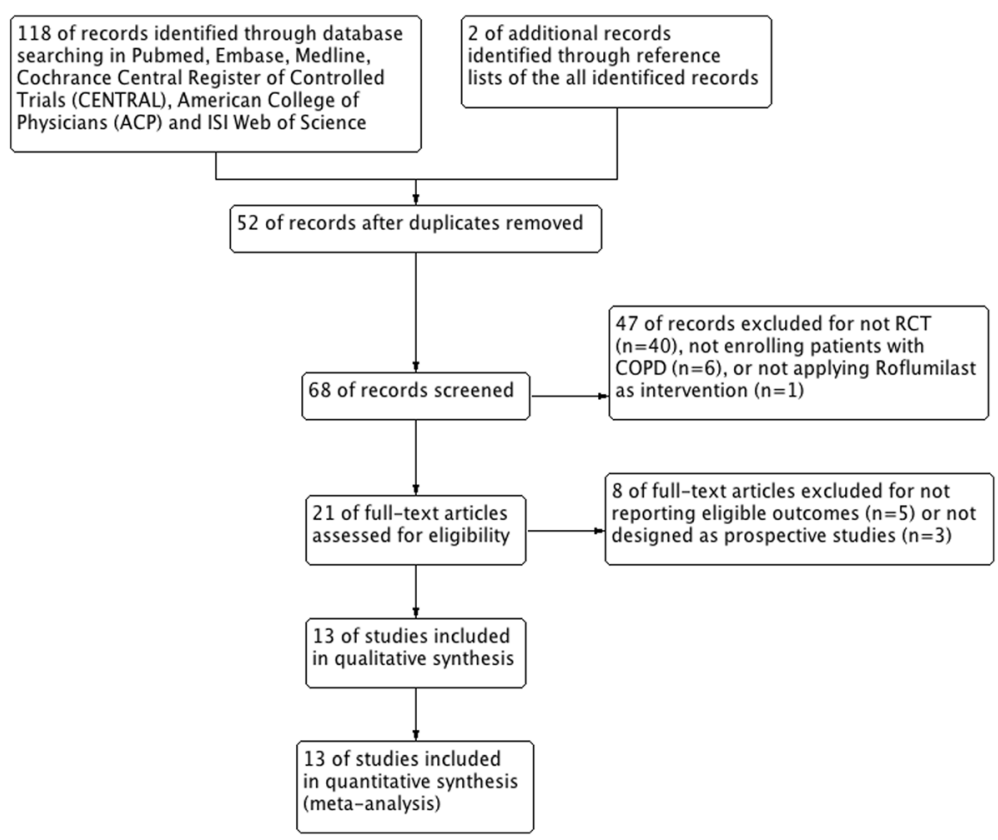

Fig. 1 Study flow diagram. ACP, American College of Physician; CENTRAL, Cochrane Central Register of Controlled Trials; COPD, chronic obstructive pulmonary disease; $R C T$, randomized controlled trial

$[13,19,23,24]$ presented data of TDI change, 4 studies $[11,13,18,21]$ presented data of SGRQ change, 10 studies $[11,13,18-25]$ showed incidence of COPD exacerbation, and 10 studies [11, 13, 14, 18-21, 23-25] showed incidence of adverse events. Details of patients' characteristics, intervention strategies, and outcomes were summarized in Tables 1 and 2.

A total of 14,563 patients with COPD were pooled from all the included trials in our final meta-analysis, among which 7,398 patients were assigned to receive roflumilast, while 7,165 patients were administered placebo. The majority of patients enrolled in the studies were male (64 92.6\%), and the mean age of patients ranged from 62 to 68 years old. All patients had a long smoking history, which was estimated to be at least 37 pack-years, and experienced a severe expiratory airflow obstruction with a mean post-bronchodilator predicted $\mathrm{FEV}_{1}$ less than $55 \%$. Details of baseline characteristics of patients in each enrolled study were shown in Table 3.

Quality assessment of the 13 enrolled studies showed that there was no bias in selection, attribution, or reporting, but 2 studies $[16,22]$ did not described methods used in allocation concealment and blinding of participants and outcome assessments, neither reported whether the outcome data was incomplete or selective (Fig. 2). No studies excluding for low quality or dubious decisions were found in the sensitivity analysis, and no publication bias was detected in the Funnel plot (Fig. 3).

\section{Heterogeneity}

We did not find statistical heterogeneity in pre- and post-bronchodilator $\mathrm{FEV}_{1}$ (Figs. 4 and 5), postbronchodilaotr FVC and $\mathrm{FEF}_{25-75}$ (Fig. 6), TDI (Fig. 7), or incidence of COPD exacerbation (Fig. 9 and 10); whereas significance statistical heterogeneity was found in postbronchodilator $\mathrm{FEV}_{6} \quad\left(I^{2}=58 \%, \quad X^{2}=4.77, \quad P=0.09\right)$ (Fig. 6), SGRQ ( $\left.I^{2}=63 \%, X^{2}=1.71, P=0.07\right)$ (Fig. 8), and incidence of adverse events $\left(I^{2}=94 \%, X^{2}=0.03, P<\right.$ 0.001) (Fig. 11).

\section{Outcomes \\ Lung function}

The mean difference of pre-bronchodilator $\mathrm{FEV}_{1}$ change from baseline between treatment with roflumilast and placebo in 12 weeks, 24 weeks and 52 weeks were $96.75 \mathrm{ml} \mathrm{(95 \%} \mathrm{CI} \mathrm{44.21} \mathrm{149.29),} 65.56 \mathrm{ml} \mathrm{(95 \%} \mathrm{CI}$ $51.84 \sim 79.28)$, and $45.08 \mathrm{ml}$ (95\% CI 33.64 56.52), respectively, which showed that there were significant differences correspondingly $(z=3.61, P<0.001 ; z=$ 9.37, $P<0.001$; and $z=7.72, P<0.001)$ as well as in overall effects $(z=12.47, P<0.001)$ (Fig. 4). As for change of post-bronchodilator $\mathrm{FEV}_{1}$ from baseline, we also found significant differences in 24 weeks (MD 71.63, $95 \%$ CI 57.44 85.82, $z=9.89, P<0.001)$ and 52 weeks (MD $50.41 \mathrm{ml}, 95 \%$ CI $40.46 \sim 60.35, z=$ 9.94, $P<0.001$ ), and in overall effects (MD $57.86 \mathrm{ml}$, $95 \%$ CI $49.80 \sim 69.51, z=14.07, P<0.001$ ) (Fig. 5). Meanwhile, significant improvement in change of postbronchodilator FVC (MD 90.37 ml, 95 \% CI 73.95 
Table 1 Details of each enrolled study

\begin{tabular}{|c|c|c|c|c|c|c|c|c|c|c|c|c|}
\hline \multirow{2}{*}{\multicolumn{2}{|c|}{ Author (Year) }} & \multirow[t]{2}{*}{ NCT No. } & \multirow[t]{2}{*}{ Patients } & \multirow[t]{2}{*}{ Population (I/C) } & \multicolumn{3}{|l|}{ Intervention } & \multirow[t]{2}{*}{ Control } & \multirow[t]{2}{*}{ Routine } & \multirow[t]{2}{*}{ Duration } & \multirow[t]{2}{*}{ Follow-up } & \multirow[t]{2}{*}{ Outcomes $^{a}$} \\
\hline & & & & & Drug & Dose & Frequency & & & & & \\
\hline \multicolumn{2}{|c|}{$\begin{array}{l}\text { Bredenbroker, } 2002 \\
\text { [16] }\end{array}$} & FK1 101 & Mild to severe COPD & $\begin{array}{l}341 \\
(169 / 172)\end{array}$ & Roflumilast & 500 ug & $\begin{array}{l}\text { Once } \\
\text { daily }\end{array}$ & Placebo & Oral & 26 weeks & 26 weeks & (1) \\
\hline \multicolumn{2}{|c|}{$\begin{array}{l}\text { Boszormenyi-Nagy, } \\
2005 \text { [17] }\end{array}$} & FK1 103 & Mild to severe COPD & $\begin{array}{l}386 \\
(200 / 186)\end{array}$ & Roflumilast & 500ug & $\begin{array}{l}\text { Once } \\
\text { daily }\end{array}$ & $\begin{array}{l}\text { Roflumilast + } \\
\text { Placebo }\end{array}$ & Oral & 24 weeks & 24 weeks & (1) \\
\hline \multicolumn{2}{|c|}{ Rabe, 2005 [11] } & M2-107 & $\begin{array}{l}\text { COPD with postbronchodilator } \mathrm{FEV}_{1} \% \\
\text { of } 30 \sim 80 \% \text {, age } \geq 40 \text { years and a } \\
\text { smoking history }>10 \text { pack-year }\end{array}$ & $\begin{array}{l}835 \\
(555 / 280)\end{array}$ & Roflumilast & 500ug & $\begin{array}{l}\text { Once } \\
\text { daily }\end{array}$ & Placebo & Oral & 24 weeks & 24 weeks & (1)(2)(3)(4)(6)(2) \\
\hline \multicolumn{2}{|c|}{ Calverley, 2007 [18] } & $\begin{array}{l}\text { NCT00430729 } \\
\text { (M2-112) }\end{array}$ & $\begin{array}{l}\text { COPD with postbronchodilator } \mathrm{FEV}_{1} \% \\
\text { of } 50 \% \text { or less, age } \geq 40 \text { years and a } \\
\text { smoking history }>10 \text { pack-year }\end{array}$ & $\begin{array}{l}1513 \\
(760 / 753)\end{array}$ & Roflumilast & 500ug & $\begin{array}{l}\text { Once } \\
\text { daily }\end{array}$ & Placebo & Oral & 52 weeks & 52 weeks & (1)(2)(3)(4)(6)(8) \\
\hline \multicolumn{2}{|c|}{ Calverley, 2009 [19] } & $\begin{array}{l}\text { NM (M2-124, } \\
\text { M2-125) }\end{array}$ & $\begin{array}{l}\text { COPD with postbronchodilator } \mathrm{FEV}_{1} \% \\
\text { of } 50 \% \text { or less, age }>40 \text { years and a } \\
\text { smoking history } \geq 20 \text { pack-year }\end{array}$ & $\begin{array}{l}3091 \\
(1537 / 1554)\end{array}$ & Roflumilast & 500 ug & $\begin{array}{l}\text { Once } \\
\text { daily }\end{array}$ & Placebo & Oral & 52 weeks & 52 weeks & (1)(2)(3)(5)(7)(9) \\
\hline \multirow[t]{2}{*}{$\begin{array}{l}\text { Fabbri, } \\
2009 \text { [13] }\end{array}$} & M2-127 & NCT00313209 & \multirow{2}{*}{$\begin{array}{l}\text { COPD with postbronchodilator } \mathrm{FEV}_{1} \% \\
\text { of } 40 \sim 70 \% \text {, age }>40 \text { years and a } \\
\text { smoking history }>10 \text { pack-year }\end{array}$} & $\begin{array}{l}933 \\
(466 / 467)\end{array}$ & $\begin{array}{l}\text { Roflumilast + } \\
\text { Salmeterol }\end{array}$ & 500ug & $\begin{array}{l}\text { Once } \\
\text { daily }\end{array}$ & $\begin{array}{l}\text { Salmeterol + } \\
\text { Placebo }\end{array}$ & Oral & 24 weeks & 24 weeks & (1)(2)(3)(7)(3) \\
\hline & M2-128 & NCT00424268 & & $\begin{array}{l}743 \\
(371 / 372)\end{array}$ & $\begin{array}{l}\text { Roflumilast + } \\
\text { Tiotropium }\end{array}$ & & & $\begin{array}{l}\text { Tiotropium + } \\
\text { Placebo }\end{array}$ & & & & (1)(2)(3)(6)(8) \\
\hline \multicolumn{2}{|l|}{$\begin{array}{l}\text { Lee, } 2011 \\
{[20]}\end{array}$} & $\begin{array}{l}\text { NCT00242320 } \\
\text { (M2-119) }\end{array}$ & $\begin{array}{l}\text { COPD with postbronchodilator } \mathrm{FEV}_{1} \% \\
\text { of } 30 \sim 80 \% \text {, age } \geq 40 \text { years and a } \\
\text { smoking history }>10 \text { pack-year }\end{array}$ & $\begin{array}{l}410 \\
(203 / 207)\end{array}$ & Roflumilast & 500ug & $\begin{array}{l}\text { Once } \\
\text { daily }\end{array}$ & Placebo & Oral & 12 weeks & 12 weeks & (1)(2)(3)(4)(5)(2) \\
\hline
\end{tabular}

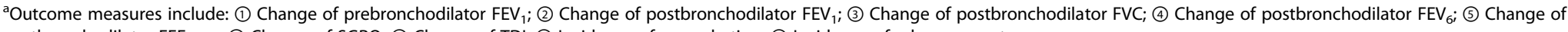
postbronchodilator $\mathrm{FEF}_{25-75}$; ( ) Change of SGRQ; (7) Change of TDI; (8) Incidence of exacerbation; (9 Incidence of adverse events

COPD chronic obstructive pulmonary disease, FEF 2575 forced expiratory flow between 25 and $75 \%$ of the vital capacity, FEV forced expiratory volume in one second, FEV ${ }_{6}$ forced expiratory volume in six seconds FVC forced vital capacity, //C intervention/control, NM not mentioned, No. numbers, SGRQ St George's Respiratory Questionnaire, TDI transition dyspnea index 
Table 2 Details of each enrolled study

\begin{tabular}{|c|c|c|c|c|c|c|c|c|c|c|c|}
\hline \multirow[t]{2}{*}{ Author (Year) } & \multirow[t]{2}{*}{ NCT No. } & \multirow[t]{2}{*}{ Patients } & \multirow[t]{2}{*}{ Population (I/C) } & \multicolumn{3}{|l|}{ Intervention } & \multirow[t]{2}{*}{ Control } & \multirow[t]{2}{*}{ Routine } & \multirow[t]{2}{*}{ Duration } & \multirow[t]{2}{*}{ Follow-up } & \multirow[t]{2}{*}{ Outcomes $^{a}$} \\
\hline & & & & Drug & Dose & Frequency & & & & & \\
\hline $\begin{array}{l}\text { Rennard, } 2011 \\
\text { [21] }\end{array}$ & $\begin{array}{l}\text { NCT00076089 } \\
\text { (M2-111) }\end{array}$ & $\begin{array}{l}\text { COPD with postbronchodilator } \mathrm{FEV}_{1} \% \\
\text { of } 50 \% \text { or less, age } \geq 40 \text { years and a } \\
\text { smoking history }>10 \text { pack-year }\end{array}$ & $\begin{array}{l}1173 \\
(567 / 606)\end{array}$ & Roflumilast & 500ug & Once daily & Placebo & Oral & 52 weeks & 52 weeks & (1)(2)(6)(9) \\
\hline $\begin{array}{l}\text { Ferguson, } 2012 \\
\text { [22] }\end{array}$ & NCT01443845 & $\begin{array}{l}\text { Severe-to-very-severe COPD patients } \\
\text { with a history of exacerbations }\end{array}$ & $2300(1150 / 1150)$ & Roflumilast & 500ug & Once daily & Placebo & Oral & 12 months & 12 months & (8) \\
\hline $\begin{array}{l}\text { O'Donnell, } 2012 \\
\text { [23] }\end{array}$ & M2-118 & $\begin{array}{l}\text { COPD with postbronchodilator } \mathrm{FEV}_{1} \% \\
\text { of } 30 \sim 80 \% \text {, age } \geq 40 \text { years and a } \\
\text { smoking history } \geq 10 \text { pack-year }\end{array}$ & $250(127 / 123)$ & Roflumilast & 500ug & Once daily & Placebo & Oral & 12 weeks & 12 weeks & (1)(2)(1)(8) \\
\hline Zheng, 2014 [24] & NCT01313494 & $\begin{array}{l}\text { COPD with postbronchodilator } \mathrm{FEV}_{1} \% \\
\text { of } 50 \% \text { or less, age } \geq 40 \text { years and a } \\
\text { smoking history } \geq 10 \text { pack-year }\end{array}$ & $626(313 / 313)$ & Roflumilast & 500ug & Once daily & Placebo & Oral & 24 weeks & 24 weeks & (1)(2)(3)(8)(9) \\
\hline $\begin{array}{l}\text { Martinez, } 2015 \\
\text { [25] }\end{array}$ & $\begin{array}{l}\text { NCT01329029 } \\
\text { (REACT) }\end{array}$ & $\begin{array}{l}\text { COPD with postbronchodilator } \mathrm{FEV}_{1} \% \\
\text { of } 50 \% \text { or less, age } \geq 40 \text { years and a } \\
\text { smoking history } \geq 20 \text { pack-year }\end{array}$ & $1935(969 / 966)$ & Roflumilast & 500ug & Once daily & Placebo & Oral & 52 weeks & 52 weeks & (2)(3)(8) \\
\hline Wells, 2015 [14] & NCT01572948 & $\begin{array}{l}\text { Moderate-to-severe COPD with } \\
\text { age }>40 \text { years and a smoking } \\
\text { history }>10 \text { pack-year }\end{array}$ & $27(11 / 16)$ & Roflumilast & 500ug & Once daily & Placebo & Oral & 30 days & $\begin{array}{l}14 \text { weeks ( } 12 \text { weeks } \\
\text { for lung function test) }\end{array}$ & (1)(2)(3) \\
\hline
\end{tabular}

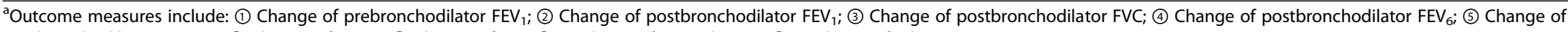

postbronchodilator $\mathrm{FEF}_{25-75}$; (6) Change of $\mathrm{SGRQ}$; (7) Change of TDI; (8) Incidence of exacerbation; (9) Incidence of adverse events

COPD chronic obstructive pulmonary disease, $F^{25} F_{25-75}$ forced expiratory flow between 25 and $75 \%$ of the vital capacity, $F E V_{1}$ forced expiratory volume in one second, $F E V_{6}$ forced expiratory volume in six seconds, $F V C$ forced vital capacity, I/C intervention/control, NM not mentioned, No. numbers, SGRQ St George's Respiratory Questionnaire, TDI transition dyspnea index 
Table 3 Baseline characteristics of patients in each enrolled trial

\begin{tabular}{|c|c|c|c|c|c|c|c|c|c|c|}
\hline \multicolumn{2}{|l|}{ Author (year) } & $\mathrm{No}^{\mathrm{a}}$ & Age $(\text { year, SD) })^{a}$ & Sex $(\text { Male, \%) })^{a}$ & $\mathrm{BMI}\left(\mathrm{kg} / \mathrm{m}^{2}, \mathrm{SD}\right)^{\mathrm{a}}$ & Smoking (Pack-year, SD) ${ }^{a}$ & Post-FEV $1 / F V C(\%, S D)^{a}$ & Post-FEV ${ }_{1}(\mathrm{~L}, \mathrm{SD})^{\mathrm{a}}$ & Post-FEV 1 (\%predicted, SD) $)^{\mathrm{a}}$ & Post-FVC $(L, S D)^{a}$ \\
\hline \multicolumn{2}{|c|}{ Bredenbroker, 2002} & 169 & NM & NM & NM & NM & NM & NM & NM & NM \\
\hline \multicolumn{2}{|c|}{$\begin{array}{l}\text { Boszormenyi-Nagy, } \\
2005\end{array}$} & 200 & NM & NM & NM & NM & NM & NM & NM & NM \\
\hline \multicolumn{2}{|l|}{ Rabe, 2005} & 555 & $64(42 \sim 87)$ & $410(75)$ & $26(5.0)$ & $41(20.6)$ & $50(12)$ & $1.50(0.48)$ & $54(13.2)$ & $3.08(0.85)$ \\
\hline \multicolumn{2}{|c|}{ Calverley, 2007} & 760 & $65(9.6)$ & $571(75)$ & $25(5.0)$ & $42(22.9)$ & $40.3(11.2)$ & $1.13(0.04)$ & $41(11.0)$ & NM \\
\hline \multicolumn{2}{|c|}{ Calverley, 2009} & 1537 & $64(9)$ & $1150(75)$ & $25.8(5.9)$ & $48(25)$ & $42.3(11.2)$ & $1.10(0.4)$ & $36.1(10.6)$ & NM \\
\hline \multirow[t]{2}{*}{ Fabbri, 2009} & M2-127 & 466 & $65(9)$ & $319(68)$ & NM & $43(22)$ & $49.8(9.4)$ & $1.51(0.4)$ & $54.7(9.1)$ & NM \\
\hline & $M 2-128$ & 371 & $64(9)$ & $262(71)$ & NM & $43(22)$ & $52.7(10.3)$ & $1.55(0.5)$ & $56.0(11.6)$ & NM \\
\hline \multicolumn{2}{|l|}{ Lee, 2011} & 203 & $68(41 \sim 91)$ & 188 (92.6) & 22.39 (3.7) & $42(22.1)$ & 50.5 (11.8) & $1.41(0.5)$ & $55.1(16.5)$ & NM \\
\hline \multicolumn{2}{|c|}{$\begin{array}{l}\text { Rennard, } 2011 \\
\text { (M2-111) }\end{array}$} & 567 & $64(8.7)$ & $387(68.3)$ & $26.0(5.7)$ & $50(28.2)$ & $43.3(10.1)$ & $1.12(0.4)$ & 36.8 (10.7) & NM \\
\hline \multicolumn{2}{|c|}{ Ferguson, 2012} & 1150 & NM & NM & NM & NM & NM & NM & NM & NM \\
\hline \multicolumn{2}{|c|}{ O'Donnell, 2012} & 127 & $60(9)$ & $93(73.2)$ & $26.4(5.0)$ & $41(20)$ & $51(11)$ & NM & $56(12)$ & NM \\
\hline \multicolumn{2}{|c|}{ Zheng, 2014} & 313 & $64.2(8.76)$ & $283(90.4)$ & $21.8(3.42)$ & $37.2(21.18)$ & 35.78 (9.69) & $0.95(0.35)$ & $36.84(11.42)$ & NM \\
\hline \multicolumn{2}{|c|}{ Martinez, 2015} & 969 & $65(8.4)$ & 718 (74) & $26.5(5.47)$ & 48 (24.6) & $40.2(10.81)$ & $1.1(0.33)$ & $35.4(9.25)$ & NM \\
\hline \multicolumn{2}{|l|}{ Wells, 2015} & 11 & $62(7)$ & 7 (64) & NM & $47(26)$ & $53(12)$ & NM & $45(12)$ & NM \\
\hline
\end{tabular}

${ }^{a}$ Data reported in all patients receiving vitamin $D$ supplementation

$B M I$ body mass index, FEV, forced expiratory volume in one second, FVC forced vital capacity, NM not mentioned, No. numbers, SD standard derivation 


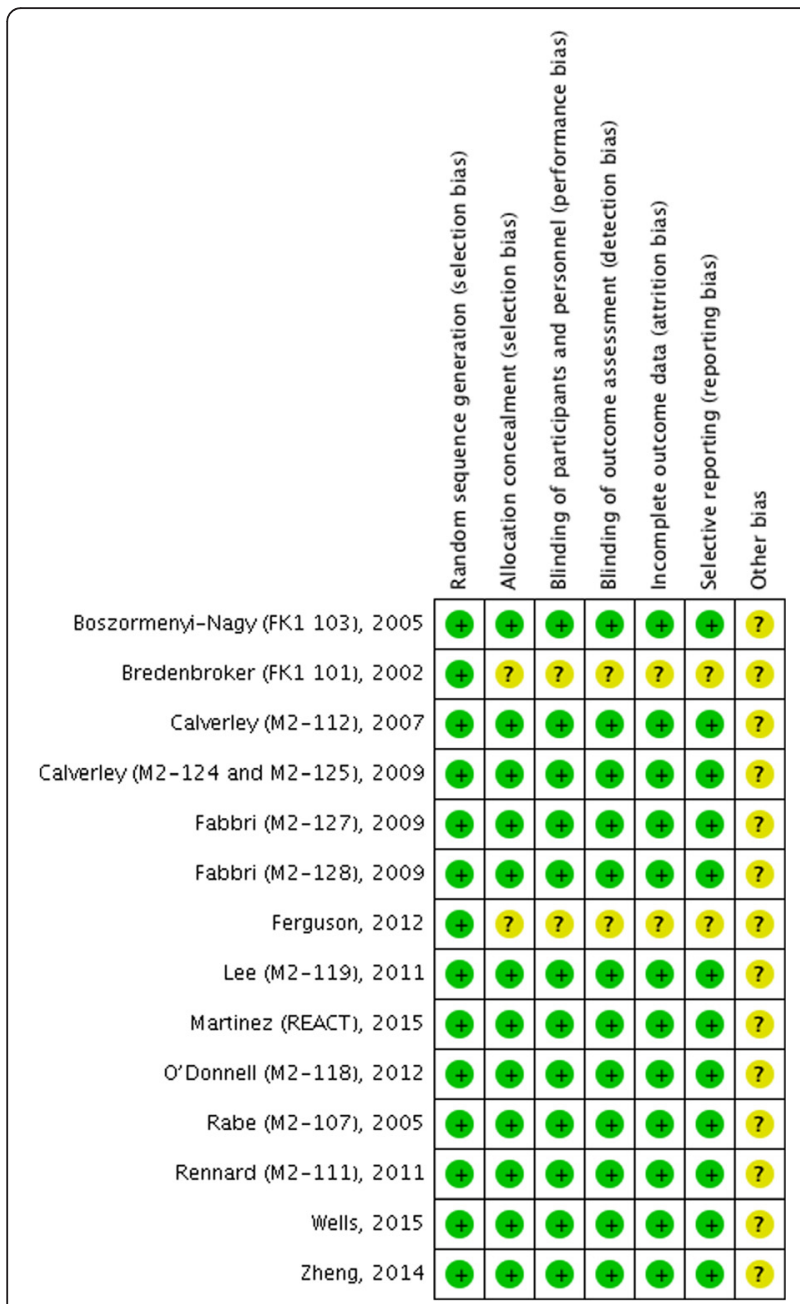

Fig. 2 Risk of bias summary

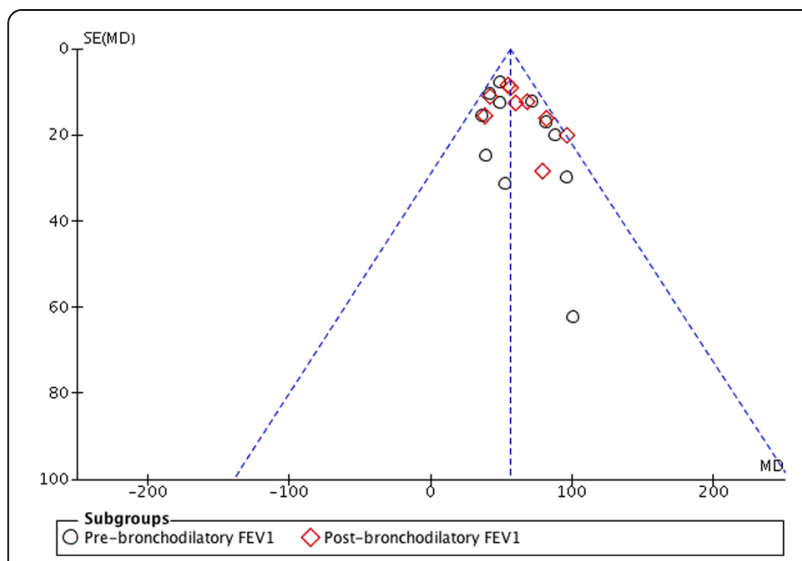

Fig. 3 Funnel plot. FEV ${ }_{1}$, forced expiratory volume in one second
106.78, $z=10.79, P<0.001$ ), $\mathrm{FEV}_{6}$ (MD $96.55 \mathrm{ml}, 95 \%$ CI $62.77 \sim 130.33, z=5.60, P<0.001)$, and $\mathrm{FEF}_{25-75}$ (MD $21.64 \mathrm{ml} / \mathrm{s}, 95 \%$ CI $12.53 \sim 30.76, z=4.65, P<$ 0.001 ) were found in roflumilast treatment compared with placebo (Fig. 6).

\section{Quality of life}

Significant improvement of TDI was detected in patients with roflumilast compared with placebo (MD 0.30, $95 \%$ CI 0.14 0.46, $z=3.67, P<0.001$ ) (Fig. 7); whereas, we did not find significant difference in SGRQ between the two treatment groups (MD -1.30, $95 \% \mathrm{CI}-3.16 \sim 0.56, z=$ 1.37, $P=0.17$ ) (Fig. 8).

\section{Incidence of COPD exacerbation}

Figures 9 and 10 displayed outcomes of incidence of COPD exacerbation in number per patient per year and in proportion, respectively, and both showed that roflumilast significantly decreased COPD exacerbation compared with placebo (MD $-0.22,95 \% \mathrm{CI}-0.30 \sim-0.14, z=5.59$, $P<0.001$; RR 0.86, $95 \%$ CI $0.81 \sim 0.91, z=5.54, P<$ $0.001)$. In addition, we also found a significant decrease of COPD exacerbation in patients with roflumilast in 24 weeks (MD $-0.42,95 \% \mathrm{CI}-0.64 \sim-0.19, z=3.68, P<$ 0.001; RR 0.80, $95 \%$ CI $0.71 \sim 0.90, z=3.62, P<0.001)$ and 52 weeks (MD $-0.19,95 \% \mathrm{CI}-0.28 \sim-0.11, z=4.59$, $P<0.001$; RR 0.88, $95 \%$ CI $0.83 \sim 0.94, z=4.18, P<$ 0.001 ), but no significance was reported in 12 weeks (RR $0.74,95 \%$ CI $0.38 \sim 1.45, z=0.87, P=0.38$ ).

\section{Incidence of adverse events}

A great variety of adverse events were detected in patients with roflumilast, among which diarrhea (2.7$12.1 \%)$, weight loss (1.0-12\%), upper respiratory tract infection (2-13.3\%), nasopharyngitis (3.4-8 \%), nausea (1.0-6 \%), and headache (1.3-4\%) were mainly reported. Discontinuations due to adverse events were more common in patients with roflumilast $(5 \sim 14 \%)$ than placebo (2.9 11\%), however, the incidence of serious adverse events resembled between patients with roflumilast ( 7 $19 \%)$ and placebo (10 $22 \%)$.

Pooled analysis of 10 studies showed that significantly higher incidence of adverse events were found in patients with roflumilast compared with placebo in 12 weeks (RR $1.51,95 \%$ CI $1.26 \sim 1.80, \mathrm{z}=4.47, \mathrm{P}<0.001)$ and 24 weeks (RR 1.76, $95 \%$ CI $1.22 \sim 2.52, \mathrm{z}=3.06, \mathrm{P}=0.002$ ), as well as in overall effects (RR 1.31, $95 \%$ CI $1.16 \sim 1.47, \mathrm{z}=4.32$, $\mathrm{P}<0.001)$. However, such a significant difference disappeared in 52 weeks (RR 1.06, $95 \%$ CI $1.00 \sim 1.11, \mathrm{z}=1.99$, $\mathrm{P}=0.05)$ (Fig. 11).

\section{Discussion}

In our meta-analysis, we found that roflumilast could significantly improve lung functions, including pre- and 


\begin{tabular}{|c|c|c|c|c|c|c|c|c|c|c|c|}
\hline \multirow{2}{*}{$\begin{array}{l}\text { Study or Subgroup } \\
1.1 .112 \text { weeks }\end{array}$} & \multicolumn{3}{|c|}{ Experimental } & \multicolumn{3}{|c|}{ Control } & \multicolumn{3}{|c|}{ Mean Difference } & \multicolumn{2}{|c|}{$\begin{array}{l}\text { Mean Difference } \\
\text { IV, Fixed, } 95 \% \mathrm{CI}\end{array}$} \\
\hline & & & & & & & & & & & \\
\hline Lee (M2-119), 2011 & 54 & 289 & 189 & -42 & 298 & 201 & $2.2 \%$ & $96.00[37.74,154.26]$ & & & \\
\hline $\begin{array}{l}\text { O'Donnell (M2-118), } 2012 \\
\text { Subtotal }(95 \% \mathrm{Cl})\end{array}$ & 70 & 479 & $\begin{array}{l}127 \\
316\end{array}$ & -30 & 501 & $\begin{array}{l}123 \\
324\end{array}$ & $\begin{array}{l}0.5 \% \\
2.7 \%\end{array}$ & $\begin{array}{r}100.00[-21.57,221.57] \\
96.75[44.21,149.29]\end{array}$ & & - & \\
\hline \multicolumn{12}{|c|}{$\begin{array}{l}\text { Heterogeneity. Chi }{ }^{2}=0.00, d f=1(P=0.95) ; 1^{2}=0 \% \\
\text { Test for overall effect: } Z=3.61(P=0.0003)\end{array}$} \\
\hline \multicolumn{12}{|l|}{ 1.1.2 24 weeks } \\
\hline Boszormenyi-Nagy (FK1 103), 2005 & 78 & 240 & 200 & 39 & 245 & 186 & $3.1 \%$ & $39.00[-9.44,87.44]$ & & & \\
\hline Fabbri (M2-127), 2009 & 39 & 192 & 456 & -10 & 194 & 463 & $11.8 \%$ & $49.00[24.04,73.96]$ & & & —- \\
\hline Fabbri (M2-128), 2009 & 65 & 229 & 365 & -16 & 229 & 364 & $6.7 \%$ & $81.00[47.75,114.25]$ & & & \\
\hline Rabe (M2-107), 2005 & 49 & 283 & 555 & -39 & 268 & 280 & $4.8 \%$ & $88.00[48.76,127.24]$ & & & \\
\hline $\begin{array}{l}\text { Zheng, } 2014 \\
\text { Subtotal }(95 \% \mathrm{Cl})\end{array}$ & 49 & 134 & $\begin{array}{r}239 \\
1815\end{array}$ & -22 & 140 & $\begin{array}{r}259 \\
1552\end{array}$ & $\begin{array}{l}12.7 \% \\
39.1 \%\end{array}$ & $\begin{array}{r}71.00[46.93,95.07] \\
65.56[51.84,79.28]\end{array}$ & & & - \\
\hline \multicolumn{12}{|c|}{$\begin{array}{l}\text { Heterogeneity. } \mathrm{Chi}^{2}=5.13, \mathrm{df}=4(\mathrm{P}=0.27) ; \mathrm{I}^{2}=22 \% \\
\text { Test for overall effect: } Z=9.37(\mathrm{P}<0.00001)\end{array}$} \\
\hline \multicolumn{12}{|l|}{ 1.1.3 26 weeks } \\
\hline $\begin{array}{l}\text { Bredenbroker (FK1 101), } 2002 \\
\text { Subtotal }(95 \% \mathrm{CI})\end{array}$ & 109 & 273 & $\begin{array}{l}169 \\
169\end{array}$ & 57 & 302 & $\begin{array}{l}172 \\
172\end{array}$ & $\begin{array}{l}2.0 \% \\
2.0 \%\end{array}$ & $\begin{array}{r}52.00[-9.08,113.08] \\
52.00[-9.08,113.08]\end{array}$ & & & \\
\hline \multicolumn{12}{|l|}{$\begin{array}{l}\text { Heterogeneity. Not applicable } \\
\text { Test for overall effect: } Z=1.67(P=0.10)\end{array}$} \\
\hline \multicolumn{12}{|l|}{ 1.1.452 weeks } \\
\hline Calverley (M2-112), 2007 & 9 & 303 & 760 & -27 & 302 & 753 & $7.9 \%$ & $36.00[5.52,66.48]$ & & & - \\
\hline Calverley (M2-124 and M2-125), 2009 & 40 & 230 & 1475 & -9 & 194 & 1511 & $31.5 \%$ & $49.00[33.72,64.28]$ & & & $\rightarrow-$ \\
\hline $\begin{array}{l}\text { Rennard (M2-111), } 2011 \\
\text { Subtotal }(95 \% \mathrm{Cl})\end{array}$ & 30 & 182 & $\begin{array}{r}545 \\
2780\end{array}$ & -12 & 178 & $\begin{array}{r}596 \\
2860\end{array}$ & $\begin{array}{l}16.8 \% \\
56.3 \%\end{array}$ & $\begin{array}{r}42.00[21.08,62.92] \\
45.08[33.64,56.52]\end{array}$ & & & $\rightarrow$ \\
\hline \multicolumn{12}{|c|}{$\begin{array}{l}\text { Heterogeneity. Chi } i^{2}=0.68, d f=2(P=0.71) ; I^{2}=0 \% \\
\text { Test for overall effect: } Z=7.72(P<0.00001)\end{array}$} \\
\hline Total $(95 \% \mathrm{Cl})$ & & & 5080 & & & 4908 & $100.0 \%$ & $54.60[46.02,63.18]$ & & & $\diamond$ \\
\hline \multicolumn{9}{|c|}{ 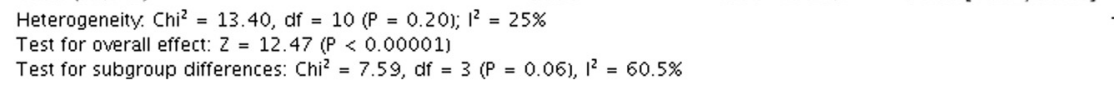 } & -200 & $\begin{array}{l}-100 \\
\text { Favours [control] }\end{array}$ & $\begin{array}{rr}100 & 2 d 0 \\
\text { Favours [experimental] }\end{array}$ \\
\hline \multicolumn{12}{|c|}{$\begin{array}{l}\text { Fig. } 4 \text { Effects of roflumilast vs. placebo on prebronchodilator } \mathrm{FEV}_{1} \text {. Cl, confidence interval; FEV } 1 \text {, forced expiratory volume in one second; SD, } \\
\text { standard derivation }\end{array}$} \\
\hline
\end{tabular}

post-bronchodilator $\mathrm{FEV}_{1}, \mathrm{FVC}, \mathrm{FEV}_{6}$ and $\mathrm{FEF}_{25-75}$, alleviate dyspnea symptoms (TDI), and decrease incidence of acute exacerbation, but could not improve quality of life (SGRQ) or decrease early acute exacerbation onset, even with a significant increase of risk of adverse events.
Persistent airflow limitation is the hallmark of COPD, which is also used to evaluate the severity and treatment responses by GOLD guideline [1]. FEV $\mathrm{FV}_{1}$ is the most common spirometric parameter to assess airflow limitation, and the post-bronchodilator $\mathrm{FEV}_{1}$ independently divided patients with COPD into 4 stages of severity, and classified

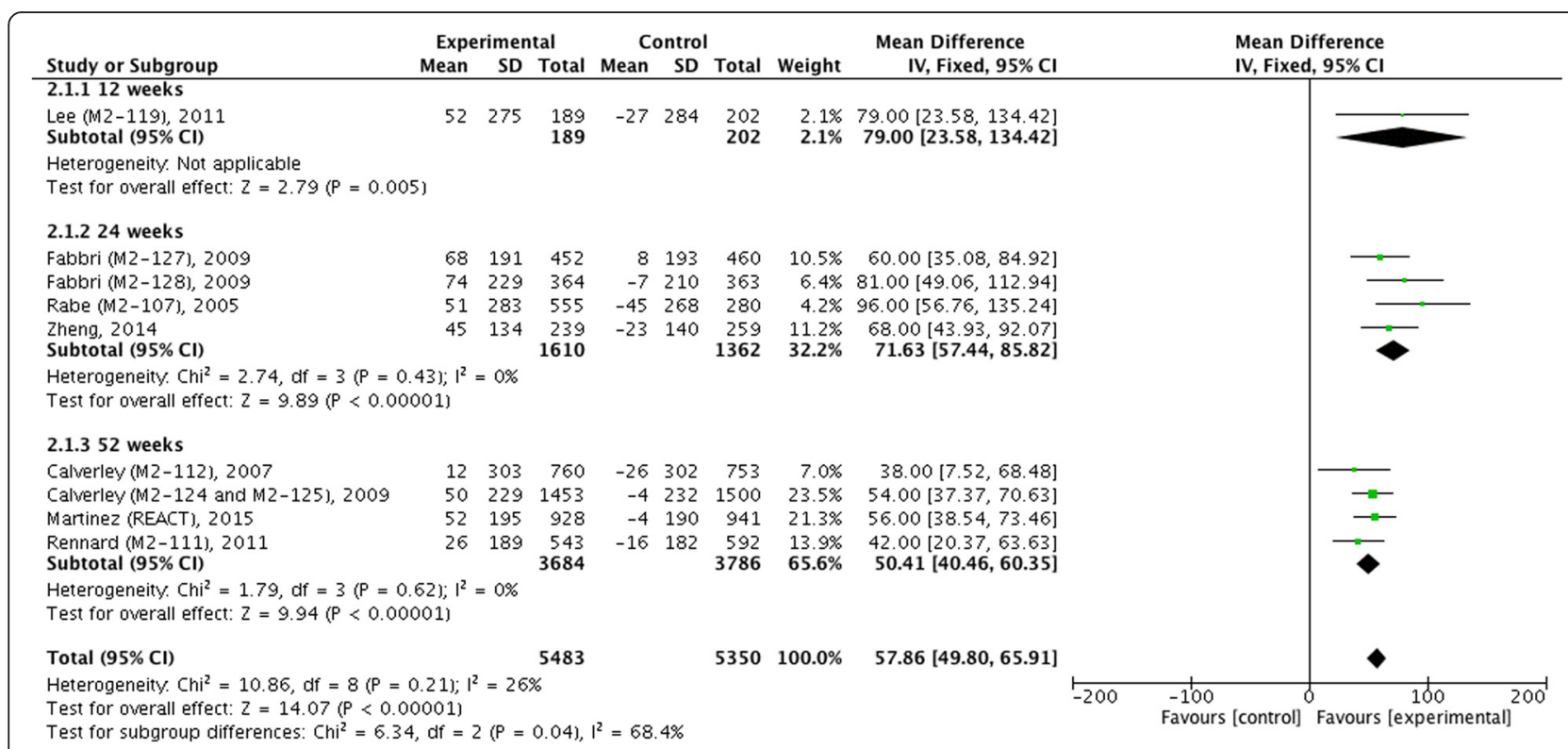

Fig. 5 Effects of roflumilast vs. placebo on postbronchodilator $\mathrm{FEV}_{1}$. Cl, confidence interval; FEV ${ }_{1}$, forced expiratory volume in one second; SD, standard derivation 


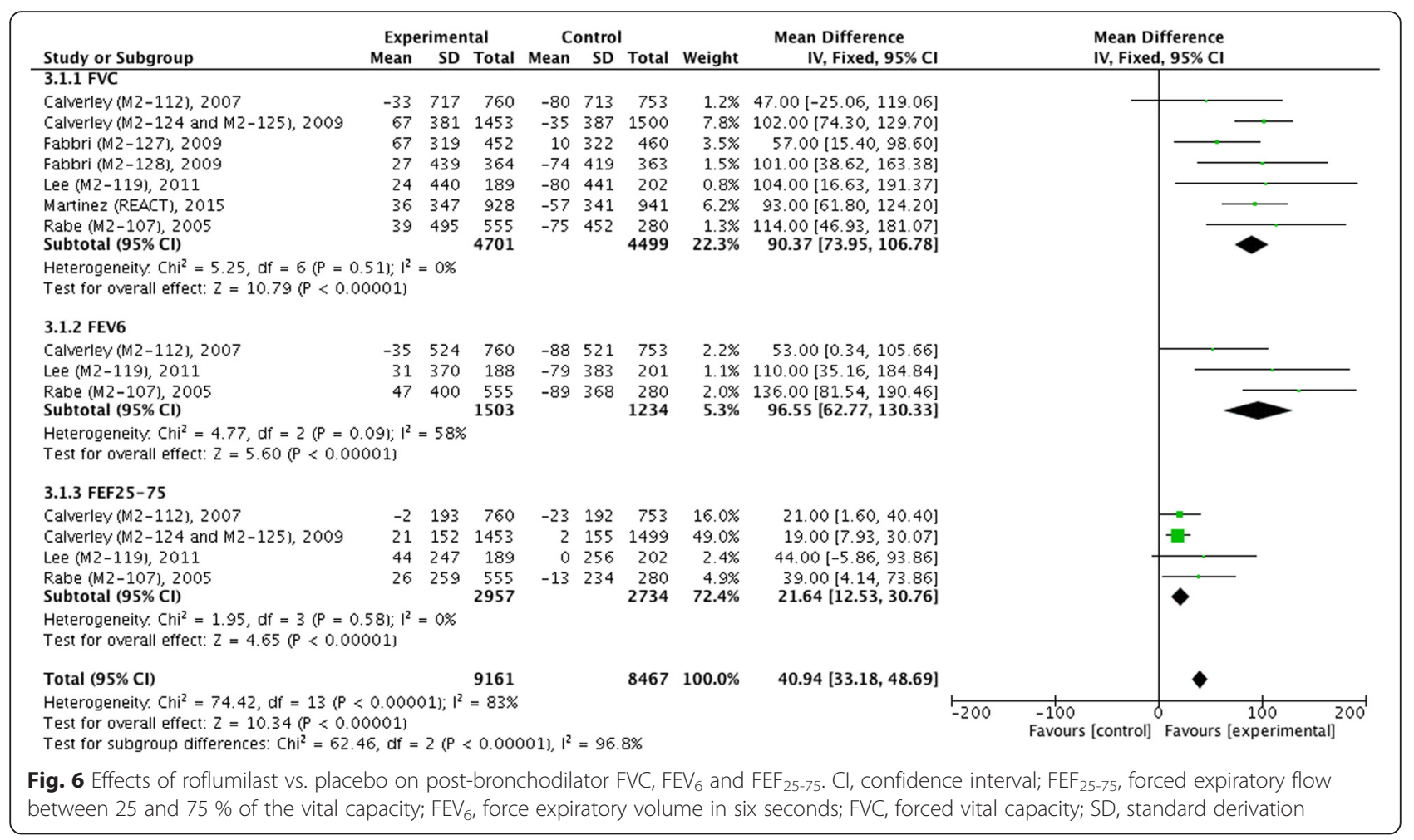

them into 4 groups with symptoms and acute exacerbations. Therefore, $\mathrm{FEV}_{1}$ improvement is usually rendered as an important factor to identify the treatment efficacy of a new drug for COPD. Previous meta-analyses have reported that roflumilast significantly improved lung function through pre-bronchodilator $\mathrm{FEV}_{1}$ compared with placebo, but they did not evaluate the post-bronchodilator $\mathrm{FEV}_{1}$ nor consider the onset time of roflumilast in improving $\mathrm{FEV}_{1}[12,26,27]$. Our study not only confirmed the effect of roflumilast on improving both preand post-bronchodilator $\mathrm{FEV}_{1}$, post-bronchodilator $\mathrm{FEV}_{6}$ and $\mathrm{FEF}_{25-75}$, but also further demonstrated that roflumilast could improve $\mathrm{FEV}_{1}$ as early as 12 weeks and the improvement effect lasted for as long as 52 weeks afterward, which we think has important clinical insights because it can facilitate physicians to set up the optimal follow-up plan and determine the administration duration.

It is well known that FVC is a volumetric parameter, which represents lung volume change and is rarely used to assess treatment responses in patients with
COPD. Recent years, however, Tashkin and his colleagues conducted a cohort study of 5,756 patients with moderate-to-severe COPD to examine acute bronchodilator responsiveness patterns in theses patients, and they found that mean improvements from baseline were $229 \mathrm{ml}$ in $\mathrm{FEV}_{1}$ and $4.07 \mathrm{ml}$ in FVC, and approximately $49 \%$ of patients with very severe COPD showed a volume response rather than a flow response to the bronchodilators [28], which also revealed the potential value of FVC alteration in evaluating treatment responses in patients with severe airflow limitation and failed to exhibit the requisite threshold increase in $\mathrm{FEV}_{1}$. In our study, 6 studies reported the change of FVC from baseline and the final pooled analysis resulted in greater improvement of FVC in patients with roflumilast than placebo. However, we could not compare the mean change of $\mathrm{FVC}$ and $\mathrm{FEV}_{1}$ or analyze the proportion of patients who responded to FVC and $\mathrm{FEV}_{1}$ due to the insufficient data reported. Therefore, future studies focusing on these issues were warranted.

Chronic and progressive dyspnea is one of the typical symptoms in patients with COPD, and is a major cause

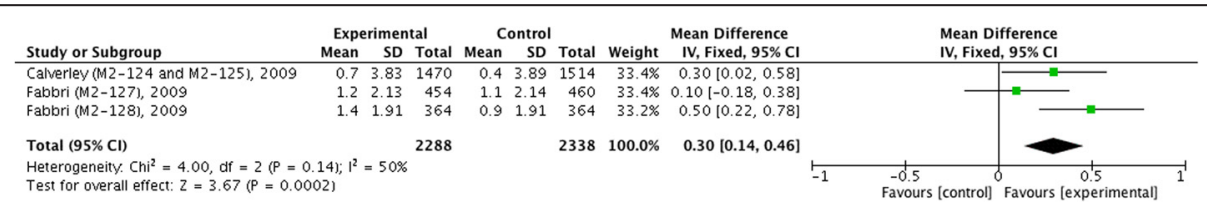

Fig. 7 Effect of roflumilast vs. placebo on TDI. Cl, confidence interval; SD, standard derivation; TDI, transition dyspnea index 


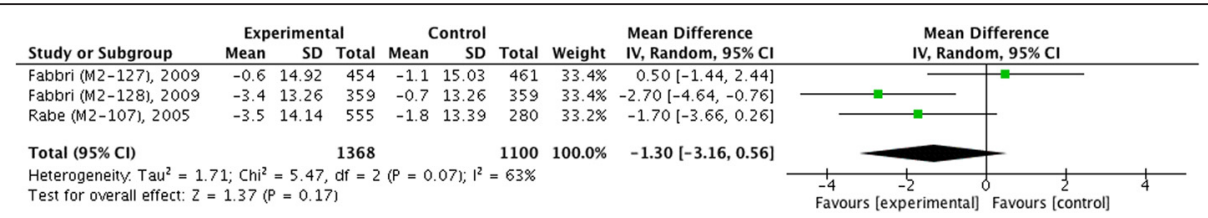

Fig. 8 Effect of roflumilast vs. placebo on SGRQ. Cl, confidence interval; SD, standard derivation; SGRQ, St George's Respiratory Questionnaire

of disability and impaired quality of life [1]. In our pooled meta-analysis, we found a significant improvement of TDI but without decreasing SGRQ scores in patients with roflumilast compared with placebo. That is, roflumilast could relieve the symptom of dyspnea, but could not attenuate other symptoms including but not limited to cough, sputum, activity endurance, and daily life. As we know, TDI is an evaluative instrument to measure breathlessness related to activities of daily living, and a large of RCTs have demonstrated reliability and accuracy in the characteristics of TDI [29]; while SGRQ is a widely used questionnaire with documented comprehensive measures, and it is recommended that regular treatment for symptoms should be considered if a COPD patient with a symptom score equivalent to SGRQ score $\geq 25$ [30-32]. Thus, the different outcomes in the effects of roflumilast on TDI and SGRQ may due to the different content and aspects in each scoring system. However, interpretation of our results should be cautious because of the potential heterogeneity in SGRQ, and the difference of corresponding parts about dyspnea in SGRQ still remains unknown.

COPD exacerbation is an acute event, which can lead to the decline of lung functions and even be fatal to patients [1]. The three meta-analyses mentioned previously also demonstrated the significant decrease of acute exacerbation rate in treatment with roflumilast, but they again did not take the different time points into account when evaluated the effect of roflumilast on affecting incidence of acute exacerbation [12, 26, 27]. Our study illustrated that although roflumilast could significantly reduce the incidence of acute exacerbation, but we did not find such an effect before 24 weeks. Therefore, the improvement of lung function may be earlier than decrease of acute exacerbation, and a minimal treatment duration of 24 weeks might be optimal to achieve improvement of both lung functions and acute exacerbation, which was also explained by a recent RCT with negative effects in $\mathrm{FEV}_{1}$ and acute exacerbation due to limited follow-up [14]. Nevertheless, we should notice the limited studies with relatively small samples and the various conditions conducted in different studies with different lengths, and further studies in evaluating acute exacerbation in 12 weeks are needed before a precise conclusion can be drawn.

It has long been recognized that weight loss, malnutrition and skeletal muscle dysfunction are common comorbidities in patients with COPD especially in later stage [33]. Based on the presently available studies, the mostly reported adverse events are diarrhea and weight loss. Our meta-analysis indeed showed a significant increase of adverse event in patients with roflumilast, which further demonstrated the conclusions by Yan and Chong [12, 27], but went contrary to the findings of Oba [26]. However, Rabe and his colleagues also perceived that most adverse events $(>90 \%)$ resolved or relieved during the course of the study [11]. Meanwhile, in our study, we also found that the incidence of adverse events

\begin{tabular}{|c|c|c|c|c|c|c|c|c|c|c|}
\hline \multirow{2}{*}{$\begin{array}{l}\text { Study or Subgroup } \\
5.1 .124 \text { weeks }\end{array}$} & \multicolumn{3}{|c|}{ Experimental } & \multicolumn{3}{|c|}{ Control } & \multicolumn{2}{|r|}{ Mean Difference } & \multicolumn{2}{|c|}{$\begin{array}{l}\text { Mean Difference } \\
\text { IV, Fixed, } 95 \% \mathrm{CI}\end{array}$} \\
\hline & & & & & & & & & & \\
\hline Fabbri (M2-127), 2009 & 1.9 & 2.92 & 466 & 2.4 & 3.86 & 467 & $3.1 \%$ & $-0.50[-0.94,-0.06]$ & & \\
\hline Fabbri (M2-128), 2009 & 1.8 & 2.77 & 371 & 2.2 & 3.24 & 372 & $3.2 \%$ & $-0.40[-0.83,0.03]$ & & \\
\hline $\begin{array}{l}\text { Rabe (M2-107), } 2005 \\
\text { Subtotal }(95 \% \mathrm{Cl})\end{array}$ & 0.75 & 1.89 & $\begin{array}{r}555 \\
1392\end{array}$ & 1.13 & 2.37 & $\begin{array}{r}280 \\
1119\end{array}$ & $\begin{array}{r}5.9 \% \\
12.3 \%\end{array}$ & $\begin{array}{l}-0.38[-0.70,-0.06] \\
-0.42[-0.64,-0.19]\end{array}$ & & \\
\hline \multicolumn{11}{|c|}{$\begin{array}{l}\text { Heterogeneity. } C h i^{2}=0.19, d f=2(P=0.91) ; l^{2}=0 \% \\
\text { Test for overall effect: } Z=3.68(P=0.0002)\end{array}$} \\
\hline \multicolumn{11}{|l|}{ 5.1.2 52 weeks } \\
\hline Calverley (M2-112), 2007 & 0.96 & 1.47 & 760 & 1.06 & 1.68 & 753 & $23.9 \%$ & $-0.10[-0.26,0.06]$ & & - \\
\hline $\begin{array}{l}\text { Calverley (M2-124 and M2-125), } 2009 \\
\text { Subtotal }(95 \% \mathrm{Cl})\end{array}$ & 1.14 & 1.3 & $\begin{array}{l}1537 \\
2297\end{array}$ & 1.37 & 1.46 & $\begin{array}{l}1554 \\
2307\end{array}$ & $\begin{array}{l}63.8 \% \\
87.7 \%\end{array}$ & $\begin{array}{l}-0.23[-0.33,-0.13] \\
-0.19[-0.28,-0.11]\end{array}$ & & \\
\hline \multicolumn{11}{|c|}{$\begin{array}{l}\text { Heterogeneity. } \mathrm{Chi}^{2}=1.86, \mathrm{df}=1(\mathrm{P}=0.17) ; \mathrm{I}^{2}=46 \% \\
\text { Test for overall effect: } \mathrm{Z}=4.59(\mathrm{P}<0.00001)\end{array}$} \\
\hline Total $(95 \% \mathrm{Cl})$ & & & 3689 & & & 3426 & $100.0 \%$ & $-0.22[-0.30,-0.14]$ & & \\
\hline \multicolumn{9}{|c|}{$\begin{array}{l}\text { Heterogeneity, } C \mathrm{Ch}^{2}=5.41, \mathrm{df}=4(\mathrm{P}=0.25) ; \mathrm{I}^{2}=26 \% \\
\text { Test for overall effect: } Z=5.59(\mathrm{P}<0.00001) \\
\text { Test for subgroup differences: } C \mathrm{Ch}^{2}=3.35, \mathrm{df}=1(\mathrm{P}=0.07), \mathrm{I}^{2}=70.2 \%\end{array}$} & 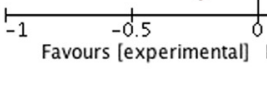 & $\begin{array}{c}0.5 \\
\text { Favours [control] }\end{array}$ \\
\hline \multicolumn{11}{|c|}{$\begin{array}{l}\text { Fig. } 9 \text { Effect of roflumilast vs. placebo on incidence of COPD exacerbation (number per patient per year). Cl, confidence interval; COPD, chronic } \\
\text { obstructive pulmonary disease; SD, standard derivation }\end{array}$} \\
\hline
\end{tabular}




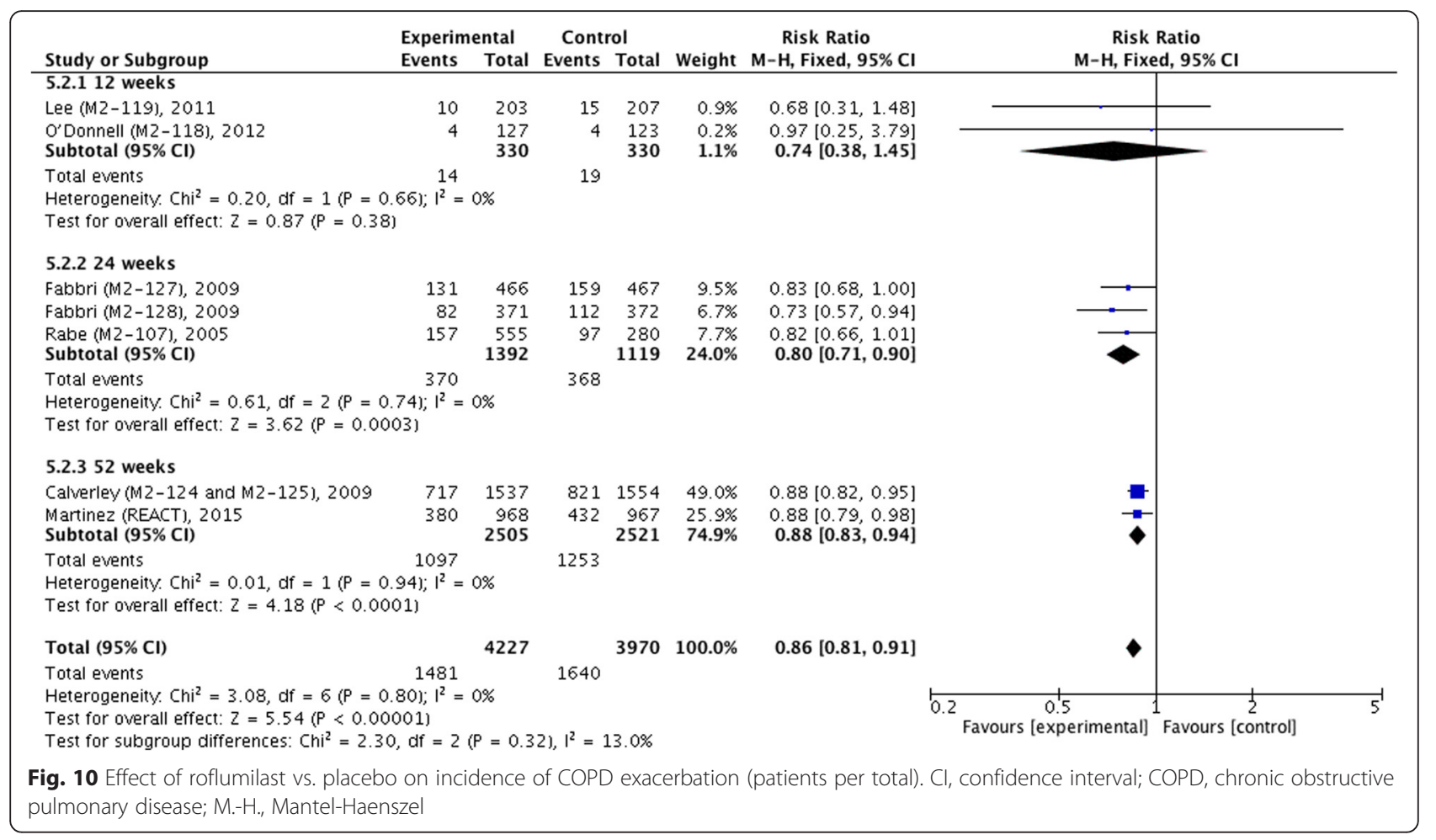

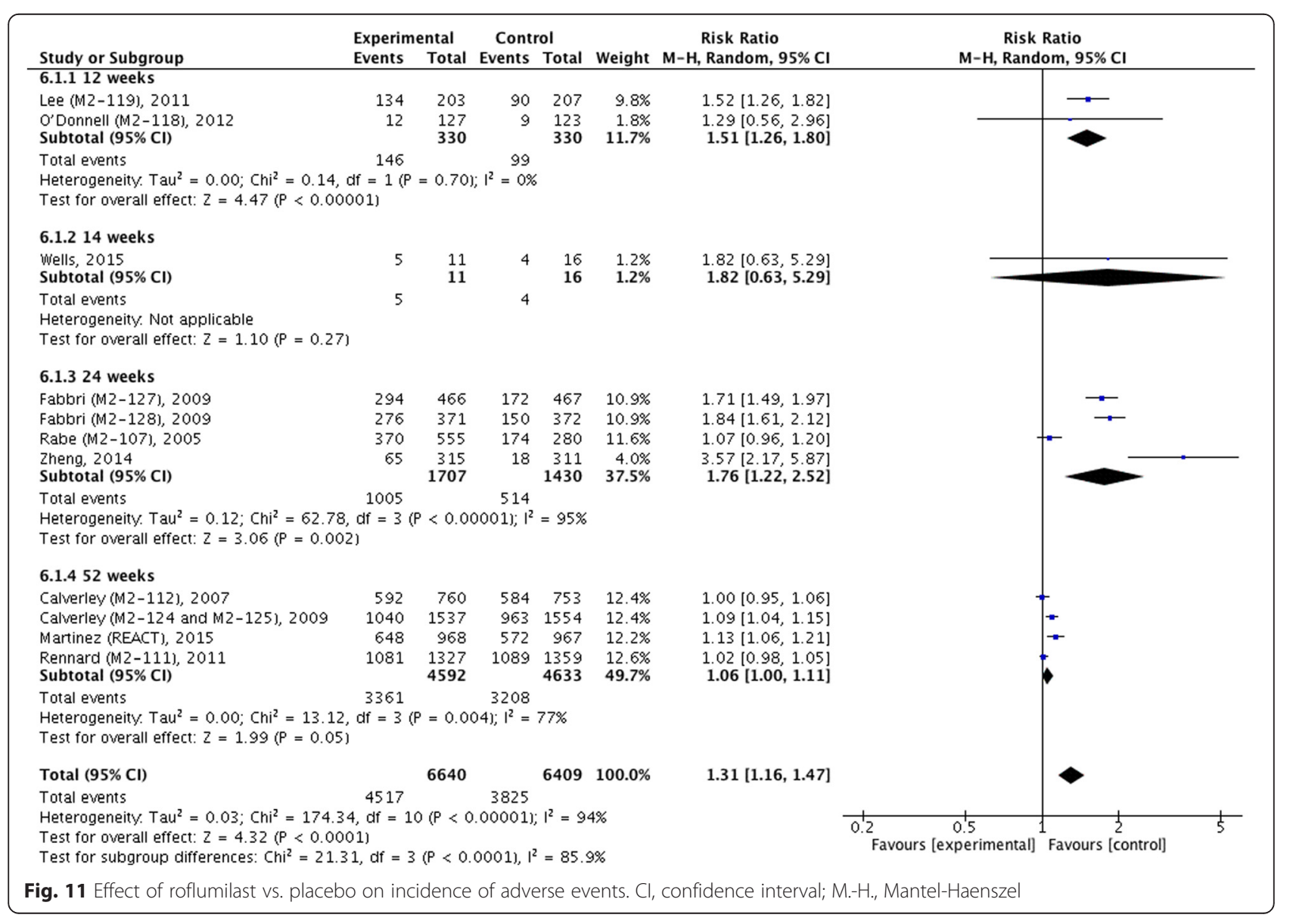


between roflumilast and placebo was similar in 52 weeks even though the $P$ value was on the borderline. As a result, administration of roflumilast should be cautious with consideration of the treatment benefits and detriments in patients with COPD comorbidities.

Limitations for our meta-analysis are as follows: First, the baseline demographics of patients and extent of airflow limitation in COPD were not identical among the enrolled trials, which may lead to selection biases. Second, potential heterogeneities existed in some outcomes such as post-bronchodilator $\mathrm{FEV}_{6}$, SGRQ and incidence of adverse events, which may cause potential confusions to our results and conclusions. Third, the number of studies and patients for pre- and post-FEV $\mathrm{F}_{1}$ varied in different follow-up groups, especially in 12 weeks, which may also result in inaccurate conclusions. Finally, subgroup analysis of FVC by follow-up time points and comparison of improvement degrees and proportions between $\mathrm{FVC}$ and $\mathrm{FEV}_{1}$ could not be achieved due to limited data and studies.

\section{Conclusions}

Roflumilast can be considered as an alternative therapy in selective patients with moderate-to-severe COPD due to the effect of lung function improvement, dyspnea alleviation and acute exacerbation decrease but increase of risk of adverse events. More large studies are needed, particularly with different follow-up and treatment duration, to further determine the role of roflumilast, including cost-effectiveness and time-to-survive, in patients with moderate-to-severe COPD.

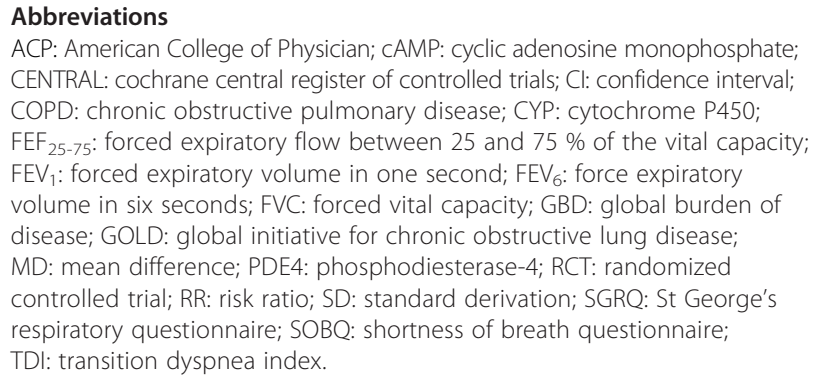

ACP: American College of Physician; CAMP: cyclic adenosine monophosphate; CENTRAL: cochrane central register of controlled trials; Cl: confidence interval; COPD: chronic obstructive pulmonary disease; CYP: cytochrome P450; $\mathrm{FEF}_{25-75}$ : forced expiratory flow between 25 and $75 \%$ of the vital capacity; $\mathrm{FEV}_{1}$ : forced expiratory volume in one second; $\mathrm{FEV}_{6}$ : force expiratory volume in six seconds; FVC: forced vital capacity; GBD: global burden of disease; GOLD: global initiative for chronic obstructive lung disease; MD: mean difference; PDE4: phosphodiesterase-4; RCT: randomized controlled trial; RR: risk ratio; SD: standard derivation; SGRQ: St George's respiratory questionnaire; $\mathrm{SOBQ}$ : shortness of breath questionnaire; TDI: transition dyspnea index.

\section{Competing interests}

The authors declare that they have no competing interests.

\section{Authors' Contribution}

$J$ and KW designed the study, conducted the literature searching and data analysis, and drafted the manuscript; DL revised the manuscript critically for important intellectual content; B-ML and C-TL made the decision to submit the report for publication. All authors read and approved the final manuscript.

\section{Acknowledgement}

This study was partly supported by Sichuan Science and Technology Agency Grant (2014SZ0010). We thank Professor Dongtao Lin (College of Foreign Languages, Sichuan University), who is specialized in biomedical writing and editing, for copyediting this manuscript.

\section{Author details}

${ }^{1}$ Department of Respiratory and Critical Care Medicine, Sichuan University, Chengdu, China. ${ }^{2}$ Department of Critical Care Medicine, West China School of Medicine and West China Hospital, Sichuan University, Chengdu, China.

${ }^{3}$ No. 37 Guoxue Alley, Chengdu 610041, China.

Received: 11 December 2015 Accepted: 1 February 2016

Published online: 17 February 2016

\section{References}

1. Global Strategy for the Diagnosis, Management, and Prevention of Chronic Obstructive Pulmonary Disease (updated 2015). Global Initiative for Chronic Obstructive Lung Disease (GLOD). 2015. www.goldcopd.org. Accessed on February 18, 2015

2. Menezes AM, Perez-Padilla R, Jardim JR, Muiño A, Lopez MV, Valdivia G, et al. Chronic obstructive pulmonary disease in five Latin American cities (the PLATINO study): a prevalence study. Lancet. 2005;366:1875-81.

3. Vos T, Flaxman AD, Naghavi M, Lozano R, Michaud C, Ezzati M, et al. Years lived with disability (YLDs) for 1160 sequelae of 289 diseases and injuries 1990-2010: a systematic analysis for the Global Burden of Disease Study 2010. Lancet. 2012;380:2163-96.

4. Mathers CD, Loncar D. Projections of global mortality and burden of disease from 2002 to 2030. PLoS Med. 2006;3, e442.

5. Kessler R, Ståhl E, Vogelmeier C, Haughney J, Trudeau E, Löfdahl CG, et al. Patient understanding, detection, and experience of COPD exacerbations: an observational, interview-based study. Chest. 2006;130:133-42.

6. Donaldson GC, Seemungal TA, Bhowmik A, Wedzicha JA. Relationship between exacerbation frequency and lung function decline in chronic obstructive pulmonary disease. Thorax. 2002;57:847-52.

7. Connors Jr AF, Dawson NV, Thomas C, Harrell Jr FE, Desbiens N, Fulkerson WJ, et al. Outcomes following acute exacerbation of severe chronic obstructive lung disease. The SUPPORT investigators (Study to Understand Prognoses and Preferences for Outcomes and Risks of Treatments). Am J Respir Crit Care Med. 1996;154:959-67.

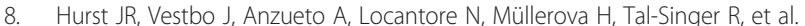
Susceptibility to exacerbation in chronic obstructive pulmonary disease. $\mathrm{N}$ Engl J Med. 2010;363:1128-38.

9. Torphy TJ. Phosphodiesterase isozymes: molecular targets for novel antiasthma agents. Am J Respir Crit Care Med. 1998;157:351-70.

10. Pinner NA, Hamilton LA, Hughes A. Roflumilast: a phosphodiesterase-4 inhibitor for the treatment of severe chronic obstructive pulmonary disease. Clin Ther. 2012;34:56-66.

11. Rabe KF, Bateman ED, O'Donnell D, Witte S, Bredenbröker D, Bethke TD. Roflumilast-an oral anti-inflammatory treatment for chronic obstructive pulmonary disease: a randomised controlled trial. Lancet. 2005;366:563-71.

12. Yan JH, Gu WJ, Pan L. Efficacy and safety of roflumilast in patients with stable chronic obstructive pulmonary disease: a meta-analysis. Pulm Pharmacol Ther. 2014:27:83-9.

13. Fabbri LM, Calverley PM, Izquierdo-Alonso JL, Bundschuh DS, Brose M, Martinez FJ, et al. Roflumilast in moderate-to-severe chronic obstructive pulmonary disease treated with longacting bronchodilators: two randomised clinical trials. Lancet. 2009;374:695-703.

14. Wells JM, Jackson PL, Viera L, Bhatt SP, Gautney J, Handley G, et al. A randomized, placebo-controlled trial of roflumilast. Effect on proline-glycineproline and neutrophilic inflammation in chronic obstructive pulmonary disease. Am J Respir Crit Care Med. 2015;192:934-42.

15. Higgins JP, Green S. eds. Cochrane Handbook for Systematic Reviews of Interventions Version 5.1.0. Oxford: The Cochrane Collaboration, 2011. Updated March 2011. www.cochrane-handbook.org. Accessed 20 March 2011

16. Bredenbroker D, Syed J, Leichtl S, Rathgeb F, Wurst W. Roflumilast, a new orally active phosphodiesterase 4 inhibitor, is effective in the treatment of chronic obstructive pulmonary disease. Eur Respir J. 2002;20:374S.

17. Boszormenyi-Nagy G, Pieters WR, Steffen H, Timar M, Vinkler I, Teichmann P, et al. The effect of roflumilast treatment and subsequent withdrawal in patients with COPD. Am J Respir Crit Care Med. 2005;169:A544.

18. Calverley PM, Sanchez-Toril F, Mclvor A, Teichmann P, Bredenbroeker D, Fabbri LM. Effect of 1-year treatment with roflumilast in severe chronic obstructive pulmonary disease. Am J Respir Crit Care Med. 2007;176:154-61.

19. Calverley PM, Rabe KF, Goehring UM, Kristiansen S, Fabbri LM, Martinez FJ. Roflumilast in symptomatic chronic obstructive pulmonary disease: two randomised clinical trials. Lancet. 2009;374:685-94. 
20. Lee SD, Hui DS, Mahayiddin AA, Roa Jr CC, Kwa KH, Goehring UM, et al. Roflumilast in Asian patients with COPD: a randomized placebo-controlled trial. Respirology. 2011;16:1249-57.

21. Rennard SI, Calverley PM, Goehring UM, Bredenbröker D, Martinez FJ. Reduction of exacerbations by the PDE4 inhibitor roflumilast-the importance of defining different subsets of patients with COPD. Respir Res. 2011;12:18.

22. Ferguson GT, Rennard SI, Hanania NA, Zhu H, Siddiqui S, Sacks H, et al. Roflumilast treatment in COPD patients taking a fixed-dose combination of long-acting beta2 agonist (LABA) and inhaled corticosteroid (ICS): Rationale and design of a prospective randomized controlled trial. Am J Respir Crit Care Med. 2012;185.

23. O'Donnell DE, Bredenbröker D, Brose M, Webb KA. Physiological effects of roflumilast at rest and during exercise in COPD. Eur Respir J. 2012;39:1104-12.

24. Zheng J, Yang J, Zhou X, Zhao L, Hui F, Wang H, et al. Roflumilast for the treatment of COPD in an Asian population: a randomized, double-blind, parallel-group study. Chest. 2014;145:44-52.

25. Martinez FJ, Calverley PM, Goehring UM, Brose M, Fabbri LM, Rabe KF. Effect of roflumilast on exacerbations in patients with severe chronic obstructive pulmonary disease uncontrolled by combination therapy (REACT): a multicentre randomised controlled trial. Lancet. 2015;385:857-66.

26. Oba Y, Lone NA. Efficacy and safety of roflumilast in patients with chronic obstructive pulmonary disease: a systematic review and meta-analysis. Ther Adv Respir Dis. 2013;7:13-24.

27. Chong J, Leung B, Poole P. Phosphodiesterase 4 inhibitors for chronic obstructive pulmonary disease. Cochrane Database Syst Rev. 2013;11, CD002309.

28. Tashkin DP, Celli B, Decramer M, Liu D, Burkhart D, Cassino C, et al. Bronchodilator responsiveness in patients with COPD. Eur Respir J. 2008; 31(4):742-50.

29. Mahler DA, Witek Jr TJ. The MCID of the transition dyspnea index is a total score of one unit. COPD. 2005;2:99-103.

30. Donohue JF, Fogarty C, Lötvall J, Mahler DA, Worth $\mathrm{H}$, Yorgancioglu A, et al. Once-daily bronchodilators for chronic obstructive pulmonary disease: indacaterol versus tiotropium. Am J Respir Crit Care Med. 2010;182:155-62

31. Jones PW, Singh D, Bateman ED, Agusti A, Lamarca R, de Miquel G, et al. Efficacy and safety of twice-daily aclidinium bromide in COPD patients: the ATTAIN study. Eur Respir J. 2012;40:830-6.

32. Vogelmeier CF, Bateman ED, Pallante J, Alagappan VK, D'Andrea P, Chen H, et al. Efficacy and safety of once-daily QVA149 compared with twice-daily salmeterol-fluticasone in patients with chronic obstructive pulmonary disease (ILLUMINATE): a randomised, double-blind, parallel group study. Lancet Respir Med. 2013;1:51-60.

33. Soriano JB, Visick GT, Muellerova H, Payvandi N, Hansell AL. Patterns of comorbidities in newly diagnosed COPD and asthma in primary care. Chest. 2005;128:2099-107.

\section{Submit your next manuscript to BioMed Central and we will help you at every step:}

- We accept pre-submission inquiries

- Our selector tool helps you to find the most relevant journal

- We provide round the clock customer support

- Convenient online submission

- Thorough peer review

- Inclusion in PubMed and all major indexing services

- Maximum visibility for your research

Submit your manuscript at www.biomedcentral.com/submit

Biomed Central 